\title{
The viability of real-time prediction and prevention of traffic accidents
}

\author{
Mohamed Abdel-Aty \& Anurag Pande
}

\begin{abstract}
This paper addresses a novel idea of real-time traffic safety improvement on freeways. First by predicting traffic crashes on the freeway mainline using on-line loop detector data, then by proposing ITS remediation strategies to reduce the crash risk in real-time. The results of the predictive models were found to be consistent with the probable mechanisms of crashes under different flow regimes. Using micro-simulation software and the crash potential determined through the models developed in this research to associate traffic characteristics with the risk of a crash occurring, several mitigation strategies were attempted to improve safety in real-time. The before (pre-ITS) and after case were then compared to examine the effects of the crash mitigation methods. The results show that Variable Speed Limits and Ramp metering have positive effects on lowering the crash potential.
\end{abstract}

\section{INTRODUCTION}

In recent years substantial growth has been observed in traffic management and information systems. The capability of storing and analyzing data has grown significantly and considerable amounts of data are being collected and stored for ITS applications. These data include speed, vehicle counts and occupancy collected from loop detectors installed beneath freeway pavement. While this growth has been around for some time, research efforts directed toward the application of freeway loop detector data for traffic safety have gained momentum only recently. Since traffic flow would be measured in terms of loop data collected in real-time, the approach diverges from traditional safety studies aimed at estimation of crash frequency or rate on freeway sections through aggregate measures of traffic flow (e.g., AADT or hourly volumes). Application of loop detector data for traffic management has been limited to incident detection algorithms. These algorithms are developed by analyzing historical post-incident loop data and attempt to detect incidents as quickly as possible. It is essentially a reactive strategy which is being rendered irrelevant with wide spread use of mobile phones and surveillance cameras. This research is an effort in the direction of proactive traffic management that would involve anticipating impending incidents such as crashes.

The problem of discriminating crash prone conditions from normal freeway traffic is set up as a classification problem in this research. To this end we also collect "non-crash" loop detector data representing 'normal' traffic conditions. Traffic parameters should then be used as inputs to the binary (crash $v s$. non-crash) on-line classification models. This research effort is aimed at the development of models that involve estimating the crash likelihood on the mainline freeway, then devising ITS strategies to reduce this likelihood.

Our group at University of Central Florida (UCF) has also been actively involved in research linking crash patterns with loop detector data. Various modeling methodologies have been explored e.g., Probabilistic neural networks (PNN) (Abdel-Aty and Pande, 2005), matched casecontrol Logistic Regression (Abdel-Aty et al., 2004), multi-layer perceptron (MLP)/radial basis 
function (RBF) neural network architectures (Pande, 2003) and Generalized Estimation Equation (Abdel-Aty and Abdalla, 2004). The data for these studies were collected from 13.2-mile central corridor of Interstate-4 in Orlando.

\section{MATCHED CASE-CONTROL LOGISTIC REGRESSION MODELS}

Loop detector data on Freeways usually include speed, vehicle counts and lane occupancy provided every 30 seconds. There have been few studies dealing with attempting to predict crashes using loop detector data. Traffic conditions measured as coefficient of variation in speed and lane occupancy have been found to be significant freeway crash precursors (e.g., Lee et al., 2002). These studies have developed crash prediction models using real-time values of these precursors obtained from underground freeway loop detectors located upstream and/or downstream of crash sites. However, these models do not take into consideration geometric and environmental factors such as horizontal curve and season. Furthermore, crash precursors are measured from loop detectors in the neighborhood of crash location at time duration prior to crashes only.

The accuracy of a real-time crash prediction model may be increased if the model utilizes information on traffic flow characteristics for both crash and non-crash cases while controlling for other external factors. This can be achieved using a within stratum analysis of a binary outcome variable $Y$ (crash or non-crash) as a function of traffic flow variables $X_{1}, X_{2}, \ldots, X_{\mathrm{p}}$ from matched crash-non-crash cases where a matched set (henceforth referred to as stratum) can be formed using crash site, time, season, day of the week, etc., so that the variability due to these factors is controlled. This is known as matched case-control study while each case refers here to a crash and control to a non-crash.

In one of our earlier studies, performed using this methodology, a logistic regression model to predict crashes on a 13-mile segment of Interstate-4 in Orlando metropolitan area was developed (Abdel-Aty et al., 2004). The model achieved satisfactory crash identification and demonstrated the feasibility of predicting crashes in real-time. The model was developed using data from a small, dense urban segment of the freeway with the crashes spanning a short period of time (seven months). The traffic, geometric and even crash characteristics on the segment remain largely uniform (i.e., same $A A D T /$ peak hour, little or no variation in the geometry along the segment, and mostly rearend crashes caused by frequent formation and dissipation of ephemeral queues). In order to develop better predictive models the crash database was expanded to include all 1528 multi-vehicle crashes that occurred during 4-year period (from 1999 through 2002) on the 36-mile instrumented corridor of Interstate-4. The corridor under consideration along with the surrounding roadways is shown in the map depicted in Figure 1. Since crashes under moderate-to-high and low speed regimes are

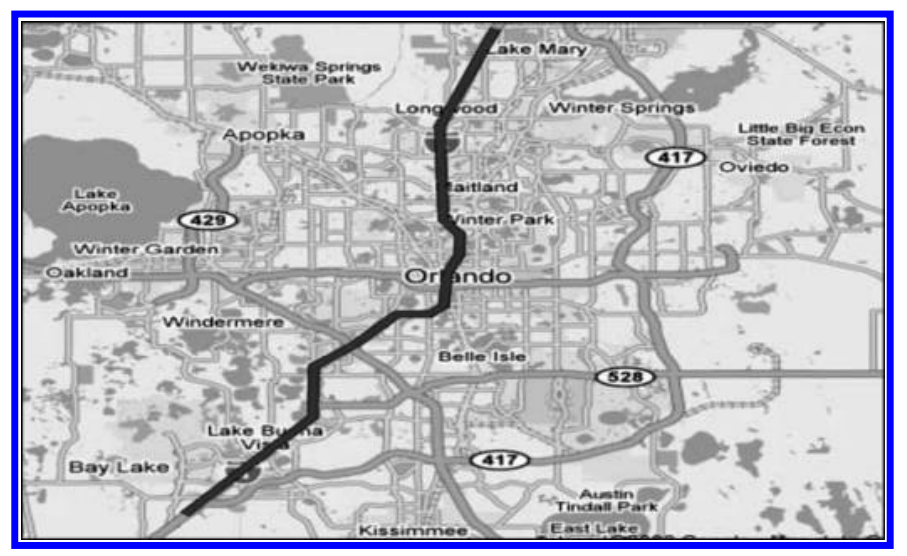

Figure 1. Map of the interstate-4 study section and adjacent roadways. 
expected to follow different occurrence mechanism they were analyzed separately using the within stratum case-control logistic regression (Abdel-Aty et al., 2005).

\section{STUDY AREA AND AVAILABLE DATA}

The following data on Interstate-4 are collected every 30 seconds; average vehicle counts, average speed, and lane detector occupancy. Sixty nine stations in each direction spaced at approximately 0.5 mile, report these parameters from the three through lanes. The crash data were collected from the FDOT crash database for the years 1999 to 2002. First, the location for all the crashes that occurred in the study area during this period was identified. For every crash, the loop detector station nearest to its location was determined and referred to as the station of crash.

\subsection{Data preparation}

Traffic data were extracted for the day of crash and on all corresponding (non-crash) days to the day of every crash to form matched sampling strata. The correspondence here means that, for example, if a crash occurred on April 12, 1999 (Monday) 6:00 PM, I-4 Eastbound and the nearest loop detector was at station 30 , data were extracted from station 30 , four loops upstream and two loops downstream of station 30 for half an hour period prior to the estimated time of the crash for all Mondays of the same season in that year at the same time. This matched sample design controls for most of the critical factors affecting crash occurrence such as driver population composition, season, day of week, location on the freeway, etc. (thus implicitly accounting for these factors). Hence, this case will have loop data table consisting of the speed, volume and lane occupancy (percent of time the loop is occupied by vehicles) values for all three lanes from the loop stations 26-32 (on eastbound direction) from 5:30 PM to 6:00 PM for all the Mondays of that season of year 1999, with one of them being the day of crash (crash case). More details of this sampling technique, application of this methodology, time of crash determination and data cleaning could be found in earlier studies by the authors (Abdel-Aty et al., 2004; 2005).

The 30-second data have random noise and is difficult to work with in a modeling framework. Therefore, the 30-second raw data were combined into 5-minute level in order to get averages and standard deviations. Thus for 5-minute aggregation half an hour period was divided into 6 time slices. The stations were named as " $B$ " to " $H$ ", with " $B$ " being farthest station upstream and so on. It should be noted that " $F$ " is the station closest to the location of the crash with " $G$ " and " $H$ " being the stations downstream of the crash location. Similarly the 5-minute intervals were also given "IDs" from 1 to 6 . The interval between time of the crash and 5 minutes prior to the crash was named as slice 1 , interval between 5 to 10 minutes prior to the crash as slice 2 , interval between 10 to 15 minutes prior to the crash as slice 3 and so on. The final dataset was created by aggregating data over all lanes and averages and standard deviations were obtained by using parameter (speed, volume and occupancy) values over the three lanes. Hence, averages (and standard deviations) at 5-minute level were based on 30 (10*3 lanes) observations. For each of the seven loop detectors $(B$ to $H)$ and six time slices (1-6) mentioned above, the values of means $(A S, A V, A O)$, standard deviations $(S S, S V, S O)$, and coefficients of variation (CVS, $\mathrm{CVV}, \mathrm{CVO})$ of speed, volume and lane occupancy, respectively, were available for all crashes and the corresponding non-crash cases. These parameters from various stations/time slices were identified with four/five letter names with each letter representing, (i) A/S/CV (average/standard deviations/coefficient of variation), (ii) $\mathrm{S} / \mathrm{V} / \mathrm{O}$ (speed/volume/occupancy), (iii) stations $\mathrm{B} / \mathrm{C} / \mathrm{D} /$ $\mathrm{E} / \mathrm{F} / \mathrm{G} / \mathrm{H}$, and (iv) time slices $1 / 2 / 3 / 4 / 5 / 6$, respectively. Due to data availability issues, there were different numbers of controls (non-crash cases) for each case (crash). To carry out matched casecontrol analysis, a symmetric data set was created (i.e., each crash case in the dataset has the same number of non-crash cases as controls) by randomly selecting five non-crash cases for each crash in the dataset. The type of crash information available in the FDOT crash database was utilized to retain only multi-vehicle crashes since the ambient traffic characteristics are more likely to affect 
crashes involving interaction among vehicles rather than the single vehicle crashes (which were removed from the dataset). The resulting dataset had 1528 matched strata available for analysis. It was ensured that the non-crash controls in the database did not experience any crash during the time data from surrounding loops was collected.

\section{PRELIMINARY ANALYSIS}

As part of preliminary data analysis, distributions of traffic parameters were explored. Figure 2 shows the histogram distribution of the variable $A S F 1$. "ASF1" is the average of speeds measured from the three lanes at the station closest to the crash location (Station F) during the 5-minute period leading to the crash (Slice 1).

The histogram distribution appears to have the shape of two adjacent approximately moundshaped distributions. The overlapping frequencies are observed over $A S F 1$ values ranging between 35 to $40 \mathrm{mph}$ (Figure 2). The two relative peaks of this histogram (approximately at 25 and 55 miles per hour) suggest the need for two separate models: One model for predicting crashes that formed the histogram with peak near $A S F 1=25$ and the other to predict crashes that formed the histogram with peak near $A S F 1=55$. The two models may be different in the sense of containing different sets of crash predictors. Hence, the value of $A S F 1=37.5 \mathrm{MPH}$ was chosen as the cut-off and two subsets of the main dataset were created. The first subset consists of all crashes with $A S F 1 \leq 37.5$ and their matched non-crash controls. The remaining crashes and their matched non-crash controls formed the second subset of data. In the two following sections the logistic regression models for the two groups of crashes are described.

\section{MODEL FOR LOW-SPEED REGIME}

The results from our previous work (Abdel-Aty et al., 2004) and some preliminary analysis (Pande and Abdel-Aty, 2005) led us to evaluate parameters from only five stations (Station $\mathrm{D}-\mathrm{H}$, two upstream stations, two downstream stations and station of the crash itself) as potential explanatory variables. It was also decided not to go beyond 10-15 minute slice (Slice 3) based on the results from the same studies (Abdel-Aty et al., 2004, Pande and Abdel-Aty, 2005). In these studies we found no evidence that the conditions 15-20 minutes prior to crash occurrences were significantly

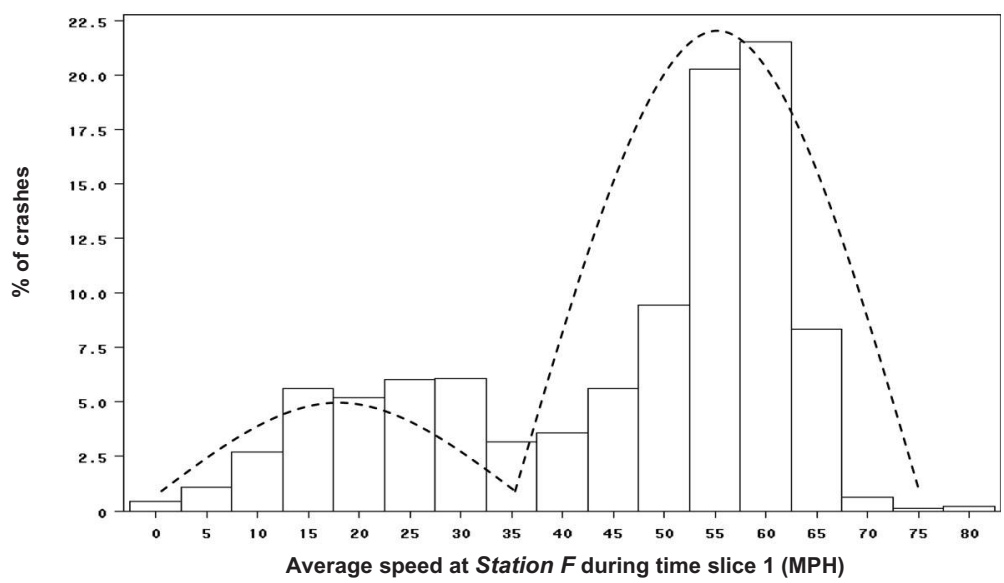

Figure 2. Distribution histogram of $A S F 1$ for all crashes. 
Table 1. Final model developed for the low-speed regime.

\begin{tabular}{lllrr}
\hline Variable & Paramete estimate & Standard error & $\chi^{2}$ & $\operatorname{Pr}>\chi^{2}$ \\
\hline LogCVSF2 & 2.64827 & 0.49216 & 28.9539 & $<.0001$ \\
Log V VSF3 & 0.88842 & 0.47859 & 3.4460 & 0.0634 \\
Log $A$ OE 2 & 1.33966 & 0.46218 & 8.4018 & 0.0037 \\
Log $A O H 3$ & 0.97766 & 0.55210 & 3.1357 & 0.0766 \\
SVF 2 & -0.43603 & 0.12289 & 12.5883 & 0.0004 \\
\hline
\end{tabular}

different than 'normal' freeway traffic. It left two time slices, Slice 2 and Slice 3 to analyze. Note that parameters from Slice 1 were not considered as an input to the model because Slice 1 is too close to the actual time of crash occurrence (and might be late anyway to try to reduce the crash risk).

The parameter estimates and related statistical summary of coefficients for the low speed regime crash prediction model is provided in Table 1. This model may be used for classification of realtime patterns under low-speed regime. Both coefficients of variation in speed (measured during time Slice 2 as well as $3 ; \log C V S F 2$ and $\log C V S F 3$ ) from the station closest to the crash location (Station $F$ ) remain in the model, with one during time Slice 2 (5-10 minutes) being much more significant. Both $\log C V S F 2$ and LogCVSF3 have positive model coefficients implying highly varying speeds (possibly due to frequent formation and dissipation of queues) prior to the crash occurrence. This high $\log C V S F 2$ is also coupled with low standard deviation in volume (indicated by negative coefficient of $S V F 2$ ) implying that the number of cars on three lanes remains fairly equal over time. The other factors in the model are $A O H 3$ and $A O E 2$, both with positive coefficients. High occupancy at one mile downstream (during 10-15 minutes Slice) and half a mile upstream (during 5-10 minutes Slice) indicates the backward propagation of congested flow regime. The interpretations (indicating congested conditions with frequent formation and dissipation of queues) largely fit into the mechanism of rear-end crashes, which are the most common type of collisions on freeways, at least under low-speed regime.

\section{MODEL FOR MODERATE-TO-HIGH SPEED REGIME}

For this model the procedures used to identify significant variables were similar to the low speed regime model but, as expected, resulted in almost entirely different set of predictors. Parameter estimates and related statistical summary of the coefficients for the high speed regime model with final set of predictors is provided in Table 2. The most interesting aspect of the model shown in Table 2 is that it does not include the coefficients of variation in speed as one of the factors. One possible reason could be that since the model is for the moderate-to-high speed regime, the coefficient of variation in speed may not be able to capture the variation in speed due to the large denominator. This led us to estimate the effect of standard deviation of speed, which also turned out to be insignificant. Another important feature of this model is that the coefficient of $\log A O F 2$ is negative indicating 'smooth' operating conditions at the station of the crash during 5-10 minutes before the crash (this is confirmed with the high average volume at Slice 3-AVE3). The only other occupancy variable entering in the model is $\log A O H 3$. With a positive coefficient it indicates some cause of congestion about one-mile downstream of the crash site (again confirmed with low $A V G 2$ and $S V H 2$ ).

It was found that the classification accuracy of the model for these conditions was inferior to the model for the low-speed crashes. The possible reason may be that in 'smoother' traffic conditions (high-speed regime) the errors on the drivers' part would contribute more significantly towards crash occurrence. Of course, there was no way to account for the pre-crash behavior of the drivers involved in historical crashes and the performance of this model suffers more due to this 
Table 2. Final model developed for the moderate-to-high-speed regime.

\begin{tabular}{lllrl}
\hline Variable & Parameter estimate & Standard error & \multicolumn{1}{c}{$\chi^{2}$} & $\operatorname{Pr}>\chi^{2}$ \\
\hline Log $A O F 2$ & -0.93423 & 0.39970 & 5.4632 & 0.0194 \\
Log $A O H 3$ & 1.14584 & 0.34156 & 11.2541 & 0.0008 \\
SVH2 & -0.22878 & 0.07181 & 10.1494 & 0.0014 \\
AVG2 & -0.10055 & 0.03376 & 8.8723 & 0.0029 \\
AVE3 & 0.05932 & 0.03419 & 3.0100 & 0.0828 \\
\hline
\end{tabular}

limitation than the low-speed model where the contributions of traffic conditions towards crash occurrence is more significant. A more detailed discussion on model building and parameter coefficient interpretations may be found in Abdel-Aty et al. (2005).

\section{MODEL BUILDING: CONCLUSIVE REMARKS}

In this section of the paper we have addressed the development of models for the identification of crash prone conditions on the freeway. The analysis of pre-crash traffic surveillance data (obtained from loop detectors) along with the matched non-crash cases is used towards that aim. The section describes the separate logistic regression models developed for crashes that occur under low-speed and moderate-to-high speed traffic conditions. These models are otherwise generic in nature as they include crashes initiated by all harmful events (rear-end, sideswipe, etc.,). Note that in some of our more recent studies (Pande and Abdel-Aty, 2006a, b) we have developed models separated by crash type as well. However, since the logistic regression models do provide us with a measure of relative risk under moderate-to-high-speed and low-speed traffic conditions, in the following sections these models are used to evaluate effectiveness of ITS strategies such as ramp metering and variable speed limits towards the reduction of real-time risk.

\section{ITS STRATEGIES TO REDUCE THE CRASH RISK}

In this study the micro-simulation software PARAMICS has been chosen to test different ITS strategies to reduce the real-time crash risk on the I-4 freeway. The use of PARAMICS to approximate variable speed limits (Lee et al., 2004) and ramp metering (Chu et al., 2004) has been successfully used in previous research. VSL is usually modeled with Variable Message Signs (VMS), which warn drivers to reduce their speed as well as changing the posted speed limits on various links as the simulation progresses. Ramp metering is also relatively simple to produce in PARAMICS using traffic signals on the freeway ramps along with complex signal phasing tools that are available.

To calibrate the freeway network, literature on similar freeway simulation models were consulted to find out the best method of determining the appropriate calibration parameters (mean headway, mean reaction time, queuing speed, and queuing distance). In most sources, the flow and travel time were used as the main factors of choice for calibration (Bertini et al., 2002; and Trapp, 2002). However, these works did not refer to the values used for the calibration parameters listed previously. Gardes et al. (2002) and Lee et al. (2002) stated their values for mean headway and driver reaction time as $1 \mathrm{~s}$ and $0.6 \mathrm{~s}$ and $0.615 \mathrm{~s}$ and $0.415 \mathrm{~s}$, respectively. This was used as the base point for our calibration procedure. Calibration was performed in order to match the locations of congestion along the freeway as well as speeds at the detector stations since these factors affect the crash likelihood equations based on the models presented above in Tables 1 and 2 . The values that produced the minimum errors in validation were a mean headway of $1.0 \mathrm{sec}, \mathrm{a}$ mean reaction time of $0.42 \mathrm{sec}$, a queuing speed of $8 \mathrm{mph}$, and a queuing distance of $8 \mathrm{ft}$. These 
parameters were close to the values that were used in other work, therefore, were considered appropriate.

\subsection{Crash likelihood}

Because of the complex nature of traffic collisions, micro-simulation software usually cannot be used to evaluate safety unless a surrogate measure is used. Abdel-Aty et al. (2005) and Pande et al. (2005) all created statistical models that can approximate the level of risk on a freeway based on the real-time traffic parameters. The models presented above to assess risk are two separate models - one to determine risk during the low-speed flow regime (speed $<37.5 \mathrm{mph}$ ) and another to determine risk during the moderate-to-high-speed flow regime (speed $\geq 37.5 \mathrm{mph}$ ). The lowspeed model is shown in (1) and involves the speed, volume, and occupancy. The moderate-tohigh-speed model, shown in (2) uses just the occupancy and volume as the input variables.

$$
\begin{aligned}
\text { Crash_Likelihood }= & 2.64827 \log C V S F 2+0.88842 \log C V S F 3+1.33966 \log A O E 2 \\
& +0.97766 \log A O H 3-0.43603 S V F 2
\end{aligned}
$$

Where $\log C V S F 2=\log$ of the standard deviation of the speed divided by the average speed $5-10$ minutes before the time of interested at the location of interest; $\log C V S F 3=\log$ of the standard deviation of the speed divided by the average speed 10-15 minutes before the time of interested at the location of interest; $\log A O E 2=\log$ of the average occupancy 5-10 minutes before the time of interest $0.5 \mathrm{mi}$ upstream of the detector of interest; $\log A O H 3=\log$ of the average occupancy 10-15 minutes before the time of interest $1 \mathrm{mi}$ downstream of the detector of interest; $S V F 2$ = Standard deviation of the speed divided by the average speed 5-10 minutes before the time of interest at the station of interest.

$$
\begin{aligned}
\text { Crash_Likelihood }= & -0.93423 \log A O F 2+1.14584 \log A O H 3-0.22878 S V H 2 \\
& -0.10055 A V G 2+0.5932 A V E 3
\end{aligned}
$$

Where $\log A O F 2=\log$ of average occupancy 5-10 minutes prior time of interest at the detector of interest; $\log A O H 3=\log$ of average occupancy $10-15$ minutes prior to time of interest $1 \mathrm{mi}$ downstream of detector of interest; $S V H 2=$ Standard deviation of volume 5-10 minutes prior to the time of interest $1 \mathrm{mi}$ downstream of the detector of interest; $A V G 2=$ Average volume 5-10 minutes prior to the time of interest $0.5 \mathrm{mi}$ downstream of the detector of interest; $A V E 3=$ Average volume 10-15 minutes prior to the time of interest $0.5 \mathrm{mi}$ upstream of the detector of interest. These models provide a measure of the likelihood of a crash occurring and how that likelihood (risk) changes over time at a particular location as the traffic flow changes. A decrease in the value given by the model over time shows a decrease in risk of a crash occurring.

\section{TESTING VARIABLE SPEED LIMITS}

Variable speed limits are typically used to reduce variances in speed on freeways (Borrough, 1997; Pilli-Sivola, 2004). By reducing the speed variability, the number of extremely short headways is reduced and the mean speed is typically lowered (Ha et al., 2003). Variable speed limits are usually implemented in order to reduce congestion and lower the average travel time. Additionally, the study by Borrough (1997) has found that VSL application also reduces the number of crashes that are observed. Borrough (1997) found that VSL implementation along with strong enforcement of the speed limits led to a reduction in crashes by almost $28 \%$ over 18 months. Although this safety benefit is not a real-time prevention measure, this reduction in crashes shows that variable speed limits have the potential to increase safety along freeways. Lee et al. (2004) performed a study in which VSL was applied to a micro-simulated network in an effort to reduce the crash potential. However, this study only focused on a 1 mile network that included only one ramp. This study, 
instead, simulates nearly a 20 mile stretch of roadway which will allow for increased flexibility of the implementation strategies that are tested. This flexibility includes the location of speed limit changes, the amount of the change and method of changing the speed limit (lowering, increasing, or simultaneous combination of the two).

\subsection{Effects of VSL on safety}

Preliminary runs involving VSL were performed by lowering the upstream speed limit near a detector for 30 minutes during the simulation. The change in the crash risk, from the before and after condition, was noted to evaluate the potential safety benefits. The preliminary runs showed that variable speed limits had little to no effect on the crash risk index during the lowspeed condition. This is most likely caused by the fact that during the low-speed scenario vehicles are traveling at congestion well below the posted speed limit and, therefore, the change in speed limit on the roadway will not effectively change the speed the vehicles are traveling at. Therefore, the analysis of variable speed limits solely focused on the moderate- to high-speed scenario where its implementation is expected to have a considerable effect on traffic flow.

The moderate- to high-speed implementation of VSL considered the upstream lowering of the speed limit, the downstream raising of the speed limit and the combination of the two. It is logical that the downstream raising of the speed limit and the upstream lowering of the speed limit together would reduce the crash risk on the freeway. Previous research (Abdel-Aty et al., $2004 ; 2005$ ) has shown that crash potential increased because of the formation of queues that create a backward forming shockwave of lower speeds while speeds were still high upstream. Therefore, in order to help dissipate these queues, the downstream speed limit was increased with the goal of moving the cars out of the queue quicker. The upstream speed limit was lowered to slow the vehicles just before the queue in order to reduce the speed variation at the shockwave interface.

For this study, a 3.25-hr simulation period was used which included a 15 minute warm-up period. To simulate the VSL strategies, speed limits were changed 30 minutes after the warm up period and were maintained for 30 minutes before reverting back to the original speed limit. The experiment included the testing of several variables related to the VSL implementation including the pattern of speed limit change, the amount of change, the location of the change, the length of the speed limit change, the progression of the change, and the gap distance between the speed limit changes. Several cases were analyzed and it was determined that a case with abrupt $15 \mathrm{mph}$ change both up and down stream showed the most significant improvement in the crash potential. This proves the effectives of the combination of upstream lowering of the speed limit and downstream raising of the speed limit over using just one method.

This scenario was then chosen to test the length of the speed limit changes. After extensive testing, the length of the changes varied from 2 to 6 miles upstream of the detector of interest and 2 to 5.5 downstream of the detector of interest. The results are about the same in each case, therefore a case where minimum change affected the freeway was chosen. Changing the speed limits 2 miles upstream and 2 miles downstream of the detector of interest affected the minimum length of roadway. Using this scenario, the progression of the change was then tested to determine the best method. This included varying the time step for the change (either an abrupt change in the speed limit or changing the speed limit in small increments every 5 or 10 minutes until the desired speed limit is achieved), the speed step (the amount of the change for each time step), and the number of time steps required to achieve the desired speed limit. All tested cases show a reduction in the crash potential but a 5 minutes time step and $5 \mathrm{mph}$ speed step proved to be the most efficient as the crash potential is lowered more in this case.

The last variable to determine the optimal VSL implementation was the gap distance between the upstream lowering of the speed limit and the downstream raising of the speed limit. The previous best method was tried using gaps of 0 to 3 miles. The results of these runs are shown in Figure 3 which is a plot of the crash potential vs. time. As shown in Figure 3, the best case found was a gap distance of 0 miles (case 1). In fact, using a gap distance of anything other than 0 miles 


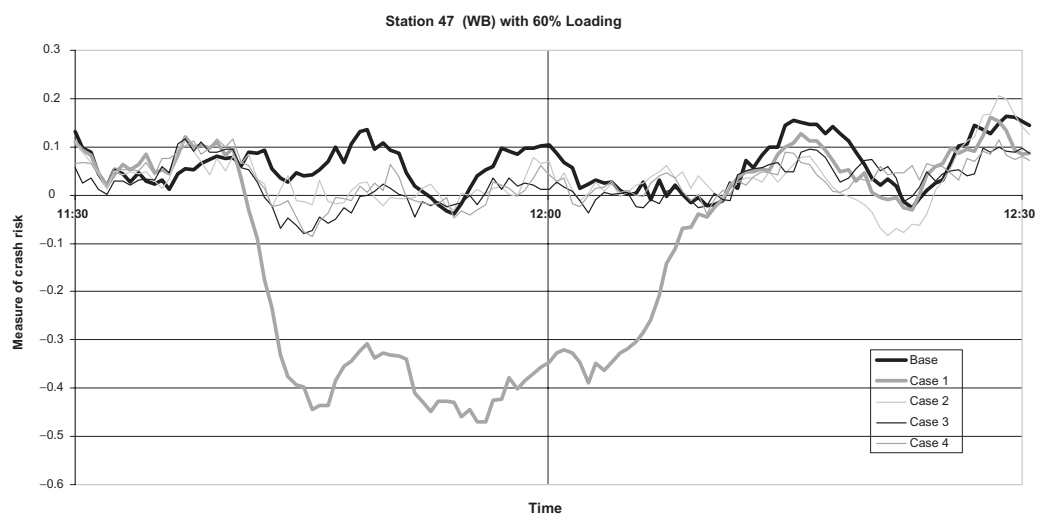

Figure 3. Crash potential vs. time at station 47 for gap distance cases.

resulted in minimal reductions in the crash risk and, therefore, would not be effective at increasing the safety on the freeway. Please note that plots similar to Figure 3 were created for each test case in order to determine which case provided the best results.

\section{TESTING RAMP METERING}

Ramp metering is typically used to limit the amount of disruption caused to the mainline traffic stream by vehicles entering from on-ramps. By allowing vehicles to enter based on the traffic conditions, vehicles are delayed at the meters but the freeway speeds remain higher (and with less variability) and the overall traffic volume increases. In fact, a study in Minnesota has shown that ramp metering increases speeds by 13 to $26 \%$ and decreases travel time by 6 to $16 \%$ (Cambridge Systematics, 2001). Currently, ramp metering is used throughout the United States in California, Minnesota, and New York, as well as in many countries throughout Europe. The signals that are used to control the ramps during ramp metering can be pre-timed (fixed) or use complex algorithms that take into account the mainline traffic flow (reactive). Fixed ramp metering limits the flow (vph) of vehicles entering the ramp, regardless of the mainline traffic situation. Reactive ramp metering takes into account the mainline flow and allows a higher rate of vehicles onto the freeway in free flow situations and reduces the flow during congestion. Though there have not been many studies done relating ramp metering to freeway safety specifically, there is plenty of evidence to show that ramp metering should have a positive safety affect. The primary purpose of ramp metering is to increase speed and, therefore, flow by reducing the speed variation on the mainline. Since the variance in speed has been found to be a significant factor in the determination of crash risk (e.g., Pande et al., 2005), any reduction in this variance should reduce the overall crash potential. Also, by implementing ramp metering, fewer extremely short headways would occur which could decrease the potential for rear-end crashes.

\subsection{Effects of ramp metering on safety}

Because variable speed limits did not show any effect at lowering the crash potential in the lowspeed situation, ramp metering was considered as a viable option. To simulate the effect of meters on ramps in PARAMICS traffic signals were placed at on-ramp entrances. Preliminary analysis involved the manual metering of ramps by visual inspection. During this process the simulation was viewed and the metered ramp was turned on and off manually based on the level of congestion that was witnessed on the mainline. After performing multiple runs of this nature, the safety benefits of ramp metering became evident. 
For the test cases, fixed ramp metering was considered. Fixed ramp metering involves using pre-timed traffic signals, at the ramp entrance to the freeway in order to control the number of vehicles that enter the mainline. By adjusting the cycle length and the green time per cycle, the rate at which vehicles are allowed to enter the freeway can be controlled. The experiment considered tested the cycle length, green time per cycle and the number of ramps that were to be metered to determine the best case scenario. Eight experimental design cases were created and are shown in Table 3.

Overall, the improvement in the crash risk was found to be minimal when metering only one ramp. Only a $3.6 \%$ decrease in the crash potential at the station of interest (station 43 ) was noted. However, at the station upstream of the ramp (station 42) an added 6.3\% decrease in crash potential was discovered. When metering is also employed on 7 ramps, the reduction in the crash potential becomes more evident. The results from one of the best cases are shown in Figure 4. These results show the summation of the crash potential throughout the length of the simulation for the stations that were affected by the ramp metering. However, both the single ramp and seven ramp best-case scenarios indicate that there is an increase in the crash potential about 3 miles upstream and downstream of station 43. Additionally, when the crash risk for a single station is examined for the duration of the ramp metering implementation at $30 \mathrm{sec}$ intervals, the best case shows that there are periods where the crash risk is actually higher than the base case. Therefore, while fixed ramp metering shows potential to mitigate the probability of crashes occurring overall, at times this type of metering increases the crash potential as well.

Table 3. Tests cases for ramp metering.

\begin{tabular}{llll}
\hline Case & Cycle length & Green time/phase & $\begin{array}{l}\text { Number of ramps } \\
\text { metered }\end{array}$ \\
\hline 1 & 50 & 25 & 1 \\
2 & 50 & 15 & 1 \\
3 & 25 & 10 & 1 \\
4 & 25 & 15 & 1 \\
5 & 50 & 25 & 7 \\
6 & 50 & 15 & 7 \\
7 & 25 & 10 & 7 \\
8 & 25 & 15 & 7 \\
\hline
\end{tabular}

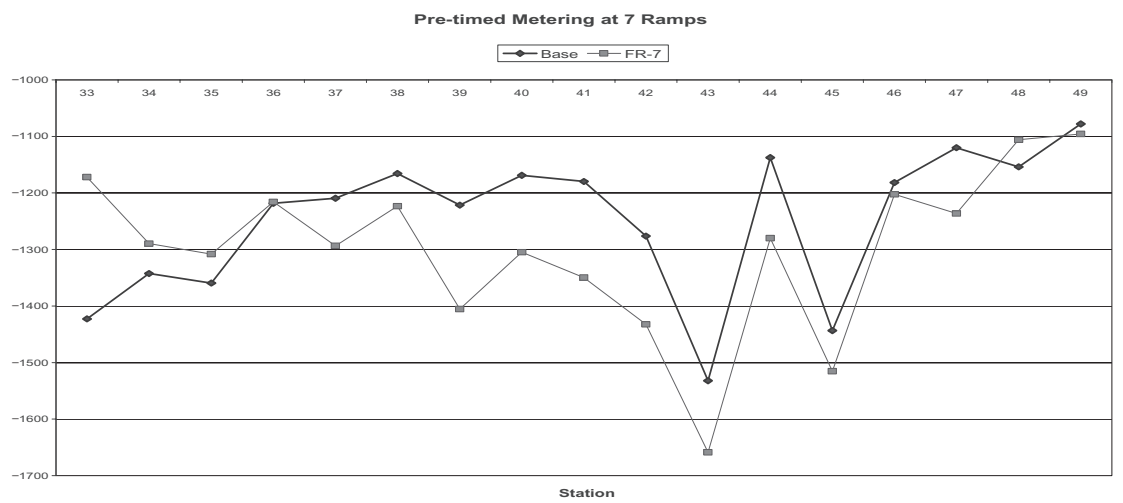

Figure 4. Crash potential vs. station for single ramp metering cases. 
However, this is most likely due to the fact that fixed ramp metering does not consider the mainline traffic when allowing vehicles to enter the freeway. Vehicles enter regardless of the congestion that exists, just at a more controlled rate than when ramp metering is not used. Therefore, in order to fully realize the potential of ramp metering as a safety measure, a feedback ramp metering system should be examined (Dhindsa, 2006). The ALINEA (Papageorgiou et al. (1991)) feedback ramp metering algorithm had been used and closely replicated the effects of manual metering by using detectors in the roadway to change the metering rate as the traffic flow changes. Dhindsa (2006) has shown increased safety benefit seen on the mainline freeway due to feedback ramp metering.

\section{CONCLUSIONS}

The results show that using the stratified case-control analysis, the log odds of crash occurrence may be obtained for a given value of certain traffic flow parameters. Hence, the potential "crash location" created due to ambient traffic conditions may be identified to warn the motorists about the impending hazard, to attempt to influence the speed to reduce its variation. The distribution of the 5-minute traffic speed measured at the station closest to the location of the crash before it occurred suggested the need for multiple models depending on the freeway operation characteristics. Two separate models were developed by splitting the whole crash data into two datasets depending on the 5-minute average speed observed just before the time of the crash. Although the procedure used in model building for low and moderate-to-high-speed models were similar, different parameters entered in the two models. This is not surprising because the crashes that occur under moderate-to-high speed traffic regime differ distinctly from their low speed counterparts not only in terms of severity but in terms of the conditions as well. The low speed crashes mostly occur in persisting congested conditions where queues form and dissipate quite frequently. In contrast, for moderate-to-high speed crashes freeway operation is usually smooth at the crash location before the crash and some disruptive conditions originating downstream and propagating backwards arguably cause drivers to make errors, thereby increasing crash potential. The time duration analyzed here is 5-15 minutes prior to the time of the crash and that's why in the moderate-to-high speed crash model more parameters from the downstream stations appear to be significant. The models developed here may be combined with the real-time application strategy to provide an effective tool to identify "real-time black-spots" on the freeway. While it is evident that the modeling technique proposed here is promising in terms of application in driver warning systems on freeways, a detailed and careful effort is required in order to study the field deployment of such a model.

In addition, this study demonstrates that the on-line mainline traffic flow data can be used as real-time surrogate measures of traffic volume on ramps and traffic conditions near ramps, and their consequent effect on the probability of ramp crashes. The findings in this study suggest the important traffic flow parameters on the mainline that should be considered in the design and management of freeway ramps to improve their safety. Although the suggestion of specific countermeasures is beyond the scope of this study, the speed management on off-ramps (e.g., in-road lights) and the warning of an impending queue using VMS on on-ramps are potential countermeasures that can be suggested based on the findings.

Finally, this study has shown significant safety benefits of using Intelligent Transportation Systems to reduce the crash likelihood on an urban freeway. The ITS measures implemented in this work, Variable Speed Limits and Ramp Metering, both help to reduce the safety risk along the mainline freeway. By simulating their effects using the PARAMICS micro-simulation, various strategies were tested to determine the best method of reducing the crash risk. For the variable speed limits, abrupt lowering of the speed limit by $15 \mathrm{mph} 2$ miles directly upstream and raising of the speed limit of $15 \mathrm{mph} 2$ miles directly downstream of the station of interest reduces the crash potential the most efficiently for moderate-to-high-speed situations (although more modest changes in speed also produced positive results). For low-speed situations, allowing vehicles to enter the 
mainline using smaller amounts of green time during shorter cycle lengths for 7 consecutive onramps in the network provides the best results. However, using a fixed ramp metering system does not react to the changing flow of traffic. Therefore, in order to fully realize the potential of ramp metering, a feedback algorithm (such as ALINEA) should be implemented to see if the reduction in crash potential can be more consistent.

\section{REFERENCES}

Abdel-Aty M. and Pande A. (2005) Identifying crash propensity using specific traffic speed conditions, Journal of Safetv Research, Vol. 36 No.1, pp. 97-108.

Abdel-Aty, M., Uddin, N., and Pande, A., (2005) Split models for predicting multi-vehicle crashes under high speed and low speed operation conditions on freeways. Transportation Research Record 1908, pp. 51-58.

Abdel-Aty, M., Uddin, N., Abdalla, F., Pande, A., and Hsia, L., (2004) Predicting freeway crashes based on loop detector data using matched case-control logistic regression. Transportation Research Record 1897, pp. 88-95.

Abdel-Aty M. and Abdalla F. (2004) Linking Roadway Geometrics and Real-Time Traffic Characteristics to Model Daytime Freeway Crashes Using Generalized Extreme Equations for Correlated Data, Journal of the Transportation Research Board, No. 1897, pp. 106-115.

Bertini, R., Lindgren, R. and Tantiyanugulchai, S. (2002) Application of PARAMICS Simulation at a Diamond Interchange," Research Report PSU-CE-TRG-02-02. Portland State University, Transportation Research Group.

Borrough, P. (1997) Variable Speed Limits Reduce Crashes Significantly in the U.K, The Urban Transportation Monitor, March 14, 1997.

Cambridge Systematics (2001) Twin Cities Ramp Meter Evaluation Phase II-Interim Ramp Meter Strategy, Report Prepared for Minnesota DOT, November 2001.

Chu, L., Liu, X. and Recker, W. (2004) Using Microscopic Simulation to Evaluate Potential Intelligent Transportation System Strategies under Non-recurrent Congestion, Transportation Research Record 1886.

Dhindsa, A. (2006) Evaluating Ramp Metering and Variable Speed Limits to Reduce Crash Potential on Congested Freeways using Micro Simulation. Master's Thesis, University of Central Florida, Orlando.

Dilmore J. (2005) Implementation Strategies for Real-Time Traffic Safety Improvements on Urban Freeways, Master's Thesis, University of Central Florida, Orlando.

Gardes, Y., May, A., Dahlgren, J. and Skabardonis, A. (2002) Freeway calibration and application of the PARAMICS model, Presented at the Transportation Research Board 81st Annual Meeting, Washington, DC.

Lee C., Hellinga B. and Saccomanno F. (2004) Assessing Safety Benefits of Variable Speed Limits, $\underline{\text { Transpor- }}$ tation Research Record 1897, pp. 183-190.

Lee, C., Saccomanno, F., and Hellinga, B., (2002) Analysis of crash precursors on instrumented freeways. Transportation Research Record 1784, pp. 1-8.

Pande A. and Abdel-Aty M. (2006a) Assessment of freeway traffic parameters leading to lane-change related collisions, Accident Analysis and Prevention (forthcoming).

Pande A. and Abdel-Aty M. (2006b) A Comprehensive Analysis of the Relationship between Real-time Traffic Surveillance data and Rear-end Crashes on Freeways, Transportation Research Record No. 1953.

Pande, A., Abdel-Aty, M., and Hsia, L., (2005) Spatio-temporal variation of risk preceding crash occurrence on freeways. Transportation Research Record 1908, pp. 26-36.

Pande A. and Abdel-Aty M. (2005) A Freeway Safety Strategy for Advanced Proactive Traffic Management, Journal of Intelligent Transportation Systems, Vol. 9 Issue 3, pp. 145-158.

Pande, A., Classification of real-time traffic speed patterns to predict crashes on freeways. MS Thesis, University of Central Florida, 2003.

Papageorgiou, M., Hadj-Salem, H., and Blosseville, J-M. (1991) ALINEA: A Local Feedback Control Law for On-Ramp Metering, Transportation Research Record 1320, pp. 58-64.

Pilli-Sivola, Y. (2000) State of the Art in Finland Concerning RWIS and Variable Message Signs," Finnish National Road Administration, February 2000.

Trapp, R. (2002) Microscopic traffic flow modeling of large urban networks - approach and techniques at the example of the City of Cologne, Transportation Research Board 81st Annual Meeting, Washington, DC. 